\title{
Erratum: Resonant-state-expansion Born approximation for waveguides with dispersion [Phys. Rev. A 93, 023835 (2016)]
}

\author{
M. B. Doost \\ (Received 9 February 2017; published 14 April 2017)
}

DOI: 10.1103/PhysRevA.95.049902

I have found a number of typographical errors in my paper that I missed in the final manuscript that was submitted for publication. They are listed below.

Equation (2) should be

$$
\left[-\nabla \times \nabla \times-\hat{\boldsymbol{\varepsilon}}_{\omega}(\mathbf{r}) \frac{1}{c^{2}} \frac{\partial^{2}}{\partial t^{2}}\right] \hat{\mathbf{E}}(\mathbf{r}, t)=0
$$

Equation (14) should be

$$
\mathcal{E}(\dot{\mathbf{r}})=-\left(k^{2}+p^{2}\right) \sum_{n} \frac{\mathbf{E}_{\mathbf{n}}(\dot{\mathbf{r}})}{2 k_{n}}\left[\frac{1}{k-k_{n}}-\frac{k}{k^{2}+p^{2}}\right] \int_{-a}^{a} \mathbf{E}_{\mathbf{n}}\left(\dot{\mathbf{r}}^{\prime}\right) \Delta \hat{\boldsymbol{\varepsilon}}\left(\dot{\mathbf{r}}^{\prime}\right) \mathcal{E}\left(\dot{\mathbf{r}}^{\prime}\right) d \dot{\mathbf{r}}^{\prime}-\sum_{n} \frac{\mathbf{E}_{\mathbf{n}}(\dot{\mathbf{r}})}{2 k_{n}\left(k-k_{n}\right)} \int_{-a}^{a} \mathbf{E}_{\mathbf{n}}\left(\dot{\mathbf{r}}^{\prime}\right) \Delta \hat{\boldsymbol{\sigma}}\left(\dot{\mathbf{r}}^{\prime}\right) \mathcal{E}\left(\dot{\mathbf{r}}^{\prime}\right) d \dot{\mathbf{r}}^{\prime}
$$

The text at the top of the right column, on page 3, should read:

Substituting this expansion into Eq. (13) and equating coefficients at the same basis functions $\mathbf{E}_{\mathbf{n}}$ results in the matrix equation. Equation (18) should be

$$
S_{n m}=\int \mathbf{E}_{\mathbf{n}}(\dot{\mathbf{r}}) \cdot \Delta \hat{\boldsymbol{\sigma}}(\dot{\mathbf{r}}) \mathbf{E}_{\mathbf{m}}(\dot{\mathbf{r}}) d \dot{\mathbf{r}}
$$

Equation (26) should be

$$
\mathcal{E}(\dot{\mathbf{r}})=-\frac{\omega^{2}}{c^{2}} \int \hat{\mathbf{G}}_{k}^{(2)}\left(\dot{\mathbf{r}}, \dot{\mathbf{r}}^{\prime}\right) \Delta \hat{\boldsymbol{\varepsilon}}\left(\dot{\mathbf{r}}^{\prime}\right) \mathcal{E}\left(\dot{\mathbf{r}}^{\prime}\right) d \dot{\mathbf{r}}^{\prime}-\frac{\omega^{2}}{c^{2}} \sum_{ \pm, j} \int G_{k}^{( \pm j)}\left(\dot{\mathbf{r}}, \dot{\mathbf{r}}^{\prime}\right) \frac{\Delta \hat{\boldsymbol{\sigma}}_{j}\left(\dot{\mathbf{r}}^{\prime}\right)}{2 k\left(k \pm \hat{q}_{j}\right)} \mathcal{E}\left(\dot{\mathbf{r}}^{\prime}\right) d \dot{\mathbf{r}}^{\prime} .
$$

The text directly below Eq. (33) should read:

which is of second order in $\varkappa_{v}$. We can write this matrix problem compactly as $\mathbf{Q}\left(\varkappa_{v}\right) \mathbf{b}_{v}=0$ with $\mathbf{Q}\left(\varkappa_{v}\right)=\varkappa_{v}^{2} \mathbf{M}+\varkappa_{v} \mathbf{C}+\mathbf{K}$. To solve this matrix problem for a basis of size $N$, I follow Ref. [14] and use the first companion linearization, defining.

The text directly below Eq. (47) should read:

I made the necessary assumption that $\hat{\boldsymbol{\varepsilon}}_{\omega}$ is a (real) symmetric matrix or a scalar so that (defined to be in this case) $\mathbf{E} \cdot \hat{\boldsymbol{\varepsilon}}_{\omega_{n}} \mathbf{E}_{n}=\mathbf{E}_{n} \cdot \hat{\boldsymbol{\varepsilon}}_{\omega_{n}} \mathbf{E}$ and nondispersive at high frequencies (see Appendices $\mathrm{E}$ and $\mathrm{F}$ ).

The text directly below Eq. (54):

The resulting RP is real and is shown in Fig. 1. This dispersion is of the form Eq. (20) and can be treated using the quadratic matrix equation Eq. (33). In order to compare this result with the one of the linear matrix equation Eq. (19), I have fitted by $\varepsilon_{\omega}$ over the wavelength range from 1.25 to $1.75 \mu \mathrm{m}$ with the form of Eq. (12), yielding.

The text at the top of the left column on p. 9 should read:

For the case of SRZ dispersion the relative error reaches the limit of about $5 \times 10^{-5}$ due to the RP approximation at $N \approx 70$. Once the perturbation method using the full Sellmeier dispersion reaches this relative error I see that upon adding further basis states more noise in the relative error plot is generated and that the average relative error changes little. Figure 9 suggests that the SRZ RSE is several orders of magnitude more efficient than the full Sellmeier RSE when considering the resonances over a small range of frequencies, such as the small range used for optical communications.

Equation (C1) should read

$$
-L=\frac{d^{2}}{d x^{2}}-p^{2}
$$

Published by the American Physical Society under the terms of the Creative Commons Attribution 3.0 License. Further distribution of this work must maintain attribution to the author(s) and the published article's title, journal citation, and DOI. 
The text directly above Eq. (D3) should read:

Assuming no degeneracy with the mode $n$, the definition of the residue tensor $\hat{\mathbf{Q}}_{n}\left(\dot{\mathbf{r}}, \dot{\mathbf{r}}^{\prime}\right)$ at a simple pole of the function $\hat{\mathbf{G}}_{k}\left(\dot{\mathbf{r}}, \dot{\mathbf{r}}^{\prime}\right)$ is.

The text directly below Eq. (D9) should read:

so we can drop terms $n \neq m$ from the summation in Eqs. (D7) and (D8) to give

The text directly below Eq. (F6) should read:

where I assume that $\hat{\boldsymbol{\varepsilon}}_{\omega}$ is a (real) symmetric matrix or a scalar so that (defined to be in this case) $\mathbf{E} \cdot \hat{\boldsymbol{\varepsilon}}_{\omega_{n}} \mathbf{E}_{n}=\mathbf{E}_{n} \cdot \hat{\boldsymbol{\varepsilon}}_{\omega_{n}} \mathbf{E}$ and so I obtain also by commutation of $\mathbf{E}$ and $\mathbf{E}_{n}$ and simple calculus in the integral shown Eq. (F6) the dispersion factor and normalization becomes.

Equation (G8) should read

$$
k\left|\dot{\mathbf{r}}-\dot{\mathbf{r}}^{\prime}\right|=k \rho-\mathbf{k}_{s} \cdot \dot{\mathbf{r}}^{\prime}+O\left(\frac{1}{\rho}\right)+\cdots
$$

Equation (G12) should read

$$
\hat{\mathbf{G}}_{k}\left(\dot{\mathbf{r}}, \dot{\mathbf{r}}^{\prime \prime}\right)=-\frac{i}{4} H_{0}\left(k\left|\dot{\mathbf{r}}-\dot{\mathbf{r}}^{\prime \prime}\right|\right) \hat{\mathbf{1}}-\omega^{2} \frac{Q e^{i k \rho}}{\sqrt{\rho \pi}} \sum_{n} \frac{\mathbf{A}_{n}\left(\mathbf{k}_{s}\right) \otimes \mathbf{E}_{n}\left(\dot{\mathbf{r}}^{\prime \prime}\right)}{2 k\left(k-k_{n}\right)} .
$$

Equation (G13) should read

$$
\hat{\mathbf{G}}_{k}\left(\dot{\mathbf{r}}, \dot{\mathbf{r}}^{\prime \prime}\right)=-\frac{i}{4} H_{0}\left(k\left|\dot{\mathbf{r}}-\dot{\mathbf{r}}^{\prime \prime}\right|\right) \hat{\mathbf{1}}-\omega^{2} \frac{Q e^{i k \rho}}{\sqrt{\rho \pi}} \sum_{n} \frac{\mathbf{A}_{n}\left(\mathbf{k}_{s}\right) \otimes \mathbf{E}_{n}\left(\dot{\mathbf{r}}^{\prime \prime}\right)}{2 k_{n}\left(k-k_{n}\right)} .
$$

